\title{
LETTER
}

\section{Stem cell transplantation}

\section{Fc-fused IL-7 mobilizes long-term HSCs in a pro-B cell-dependent manner and synergizes with G-CSF and AMD3100}

\author{
Sora Kim $\mathbb{D}^{1} \cdot$ Young-Min $\mathrm{Kim}^{2} \cdot$ Hyekang Kim $\mathbb{D}^{2} \cdot$ Yeon-Woo Kang $\mathbb{D}^{1} \cdot$ Subin Park $\mathbb{D}^{1} \cdot$ Sang-In Yang ${ }^{3}$. \\ Donghoon $\mathrm{Choi}^{4} \cdot$ Young Chul Sung $\mathbb{D}^{1,3} \cdot$ Seung-Woo Lee $\mathbb{B}^{1,2}$
}

Received: 31 August 2020 / Revised: 2 April 2021 / Accepted: 29 April 2021 / Published online: 18 May 2021

(c) The Author(s) 2021. This article is published with open access

\section{To the Editor:}

Hematopoietic stem cell (HSC) transplantation is a procedure that reestablishes hematopoietic function by infusing healthy HSCs into patients with severe hematopoietic disorders. Blood HSCs have successfully replaced traditional bone marrow (BM) transplants because of the non-invasive and safe collection process, as well as the favorable clinical outcomes following HSC transplantation [1]. Currently, recombinant human granulocyte colony-stimulating factor (rhG-CSF) is widely used to mobilize HSCs from the BM into the peripheral blood (PB) [2]. However, up to $40 \%$ mobilization failure has been reported in patients treated with G-CSF-based regimens, indicating a clinical need to develop successful therapies to induce sufficient stem cell mobilization [3].

Interestingly, it was reported that repeated administration of recombinant human interleukin-7 (rhIL-7) in mice mobilizes hematopoietic stem and progenitor cells (HSPCs) from the BM, with increases of lymphoid, myeloid, and erythroid cells in the periphery [4-6]. However, the cellular compartment of mobilized HSPCs in response to IL-7 and

These authors contributed equally: Sora Kim, Young-Min Kim

Supplementary information The online version contains supplementary material available at https://doi.org/10.1038/s41375021-01274-6.

Seung-Woo Lee

sw_lee@postech.ac.kr

1 Department of Life Sciences, Pohang University of Science and Technology, Pohang, Republic of Korea

2 Division of Integrative Biosciences and Biotechnology, Pohang University of Science and Technology, Pohang, Republic of Korea

3 Genexine, Inc., Seongnam-si, Gyeonggi-do, Republic of Korea

4 Research Institute of NeoImmunetech, Co., Itd. Bio Open Innovation Center, Pohang, Republic of Korea its underlying mechanisms remain unclear. Herein, we investigated IL-7-induced HSC mobilization using hybrid Fc-fused long-acting rhIL-7 (rhIL-7-hyFc) which has greater in vivo stability than conventional rhIL-7 [7].

To identify which fractions of HSPCs are mobilized by exogenous IL-7, we treated rhIL-7-hyFc as a single dose into wild-type (WT) mice and analyzed HSCs and progenitor subsets (short-term HSC (ST-HSC), hematopoietic progenitor cell-2 (HPC-2), and multipotent progenitor (MPP)). Following rhIL-7-hyFc injection, an increase in circulating HSPCs (phenotyped by Lineage ${ }^{-} \mathrm{Sca}-{ }^{+} \mathrm{c}-\mathrm{Kit}^{+}$, LSK) and a reduction in BM HSPCs were detected (Fig. 1a, Supplementary Fig. S1a, d, e), suggesting the mobilization of HSCs and progenitors from the BM into the periphery. HSC mobilization peaked at day 3 post-treatment and continued into day 7 in a dose-dependent manner (Fig. 1b, c, Supplementary Fig. S1b, c). HSCs mobilized by rhIL-7hyFc treatment exhibited multipotency and long-term reconstituting activity (Supplementary Fig. S1f, g). Based on our animal study, we explored whether rhIL-7-hyFcinduced mobilization of HSCs occurs in humans. We analyzed human peripheral blood mononuclear cells (PBMCs) isolated from healthy volunteers after rhIL-7-hyFc administration [8]. Results showed a significant increase in PB $\mathrm{CD}^{+}{ }^{+}$cells in the rhIL-7-hyFc-treated group compared with the placebo group (Fig. 1d, Supplementary Fig. S1h, i), demonstrating the ability of rhIL-7-hyFc to mobilize HSPCs in humans.

Given that most HSPCs do not express IL-7 receptor (IL7R) whereas mature $\mathrm{T}$ cells and developing B-cells highly express IL-7R (Supplementary Fig. S2) [9], we expected that IL-7R-expressing lymphocytes in the BM may contribute to HSC mobilization. Similar HSC mobilization was observed in recombination-activating gene 1-deficient mice (Rag-1 KO) (Fig. 1e, Supplementary Fig. S3a), demonstrating that mature $\mathrm{T}$ cells and developing pre-B and immature $\mathrm{B}$ cells are not required for IL-7-induced HSC mobilization. Following rhIL-7-hyFc treatment, pro-B cells proliferated 
a

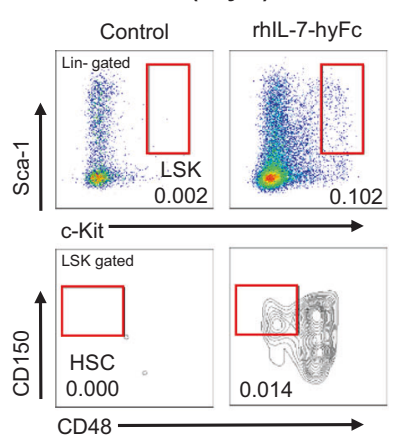

d

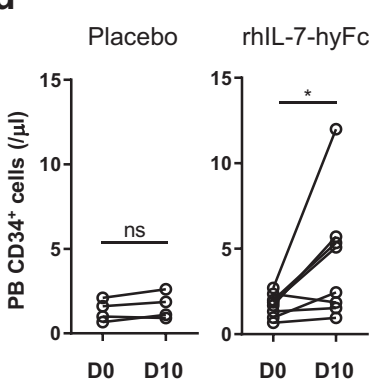

b
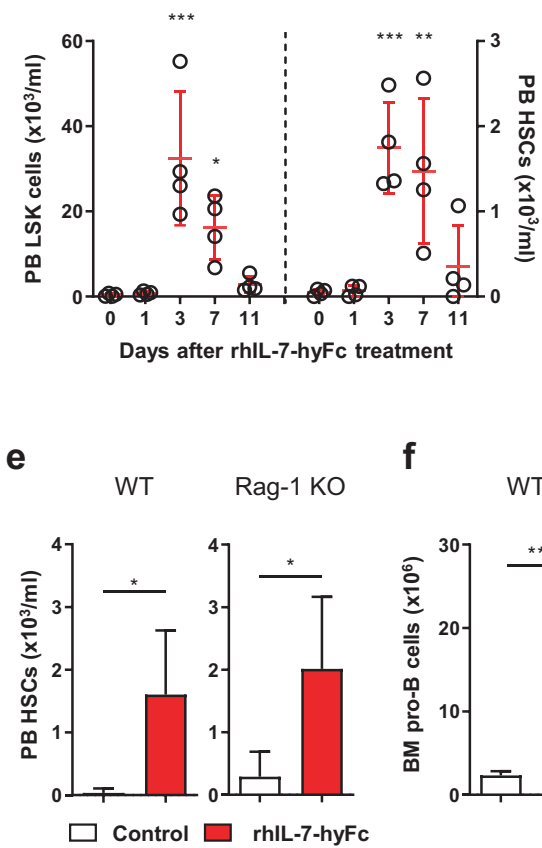

\section{f}
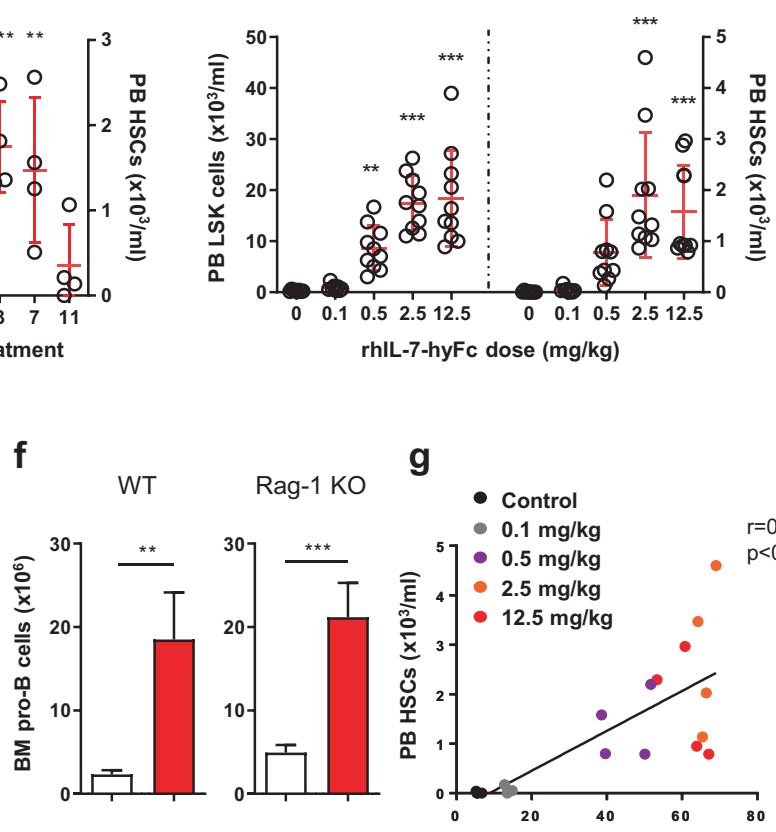

g

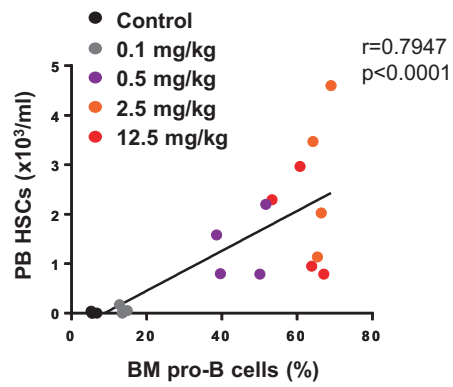

h

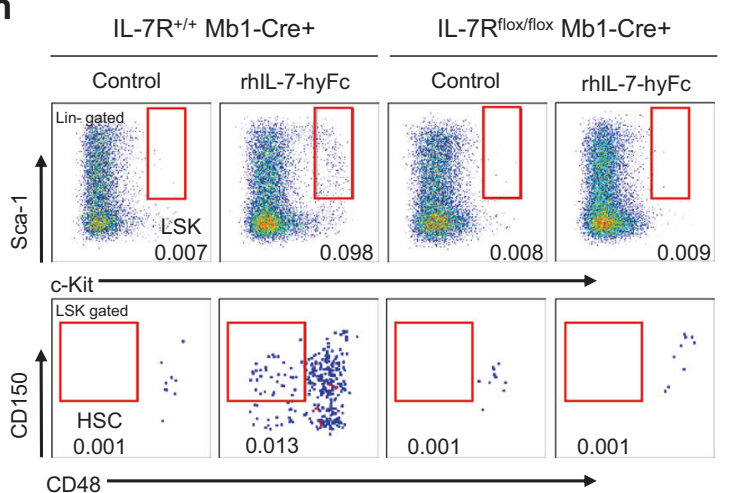

Fig. 1 Single dose administration of rhIL-7-hyFc mobilizes longterm HSCs in a pro-B cell-dependent manner. a, b Kinetics of Lineage ${ }^{-} \mathrm{Sca}-1^{+} \mathrm{c}-\mathrm{Kit}^{+}$(LSK) cell and hematopoietic stem cell (HSC) mobilization upon rhIL-7-hyFc $(2.5 \mathrm{mg} / \mathrm{kg})$. Representative dot plot at day 3 post-treatment (a) and numbers of peripheral blood (PB) LSK cells and HSCs $(\mathbf{b})(n=4)$. c Dose-dependent mobilization of LSK cells and HSCs at day 3 post-treatment $(0$ and $0.1 \mathrm{mg} / \mathrm{kg} ; n=8$, other groups; $n=10)$. d Blood CD $34^{+}$cells following the administration of single dose rhIL-7-hyFc $(60 \mu \mathrm{g} / \mathrm{kg})$ in healthy volunteers (placebo; $n=4$, rhIL-7-hyFc; $n=8$ ). e Numbers of PB HSCs mobilized by rhIL-7-hyFc treatment $(2.5 \mathrm{mg} / \mathrm{kg})$ in wild-type (WT) and recombination-activating gene 1 knockout (Rag-1 KO) mice (control;

excessively in both WT and Rag-1 KO mice, whereas prepro-B cells were largely unaffected (Fig. 1f, Supplementary Fig. S3b). Furthermore, increases in BM pro-B cells showed a strong correlation with blood HSC in response to rhIL-7hyFc (Fig. 1g), suggesting that pro-B cell responses to rhIL7-hyFc play a role in HSC mobilization. Thus, we generated IL-7 ${ }^{\text {flox/flox }} \mathrm{Mb} 1-\mathrm{Cre}+$ mice in which the IL-7R-dependent i
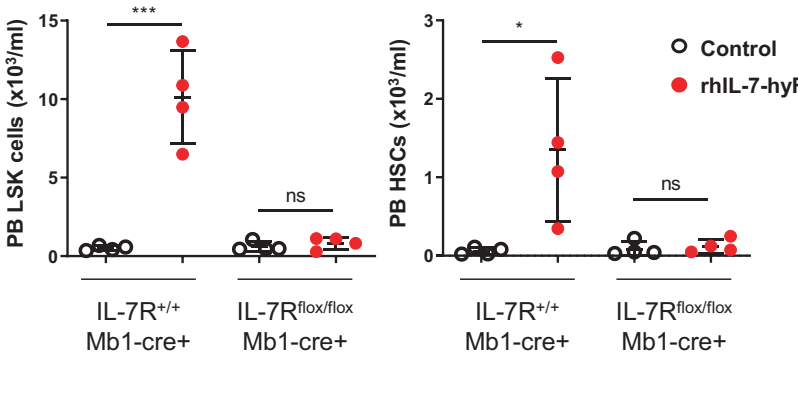

$n=4$, rhIL-7-hyFc; $n=5$ ). $\mathbf{f}$ Numbers of BM pro-B cells in WT and Rag-1 KO mice at day 3 post-rhIL-7-hyFc treatment (control; $n=4$, rhIL-7-hyFc; $n=5$ ). g Correlation between mobilized PB HSCs and BM pro-B cells at day 3 post-rhIL-7-hyFc treatment $(n=4)$. h, i Mobilization of LSK cells and HSCs by rhIL-7-hyFc treatment in IL$7 \mathrm{R}^{\text {flox/flox }} \mathrm{Mb} 1-\mathrm{Cre}+$ mice. Representative dot plot (h) and numbers of PB LSK cells and HSCs (i) $(n=4)$. Data are representative of two or three independent experiments and presented as mean \pm SD. $P$ values were determined by one-way ANOVA with Dunnett's multiple comparison for (b), (c), Wilcoxon signed-rank test for (d), unpaired $t$ test for $(\mathbf{e}),(\mathbf{f}),(\mathbf{i})$, and Spearman's correlation test for $(\mathbf{g})$. " $n$ " indicates the sample number. $* P<0.05, * * P<0.01, * * * P<0.001$.

proliferation of pro-B cells was impaired (Supplementary Fig. S3c) [10]. Contrary to $\mathrm{IL}^{-7 \mathrm{R}^{+/+}} \mathrm{Mb} 1-\mathrm{Cre}+$ control mice, rhIL-7-hyFc treatment in IL-7R ${ }^{\text {flox/flox }} \mathrm{Mb} 1-\mathrm{Cre}+$ mice did not show increased HSPCs in the PB nor reduction of BM HSPCs (Fig. 1h, i, Supplementary Fig. S3d, e). Collectively, these results demonstrate that $\mathrm{BM}$ pro-B cells are essential mediators for IL-7-induced HSC mobilization. 

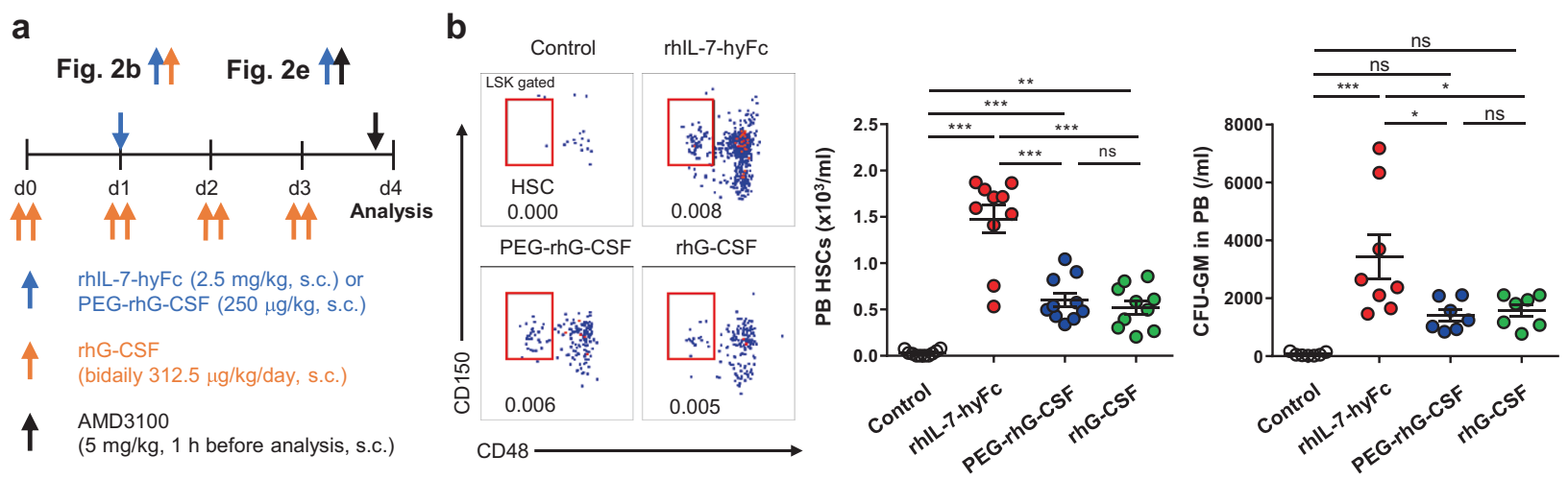

C

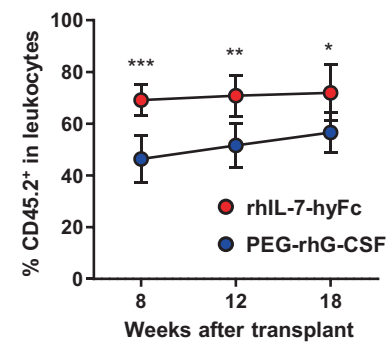

f

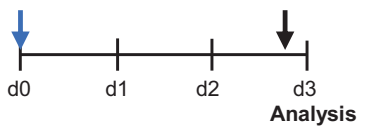

g

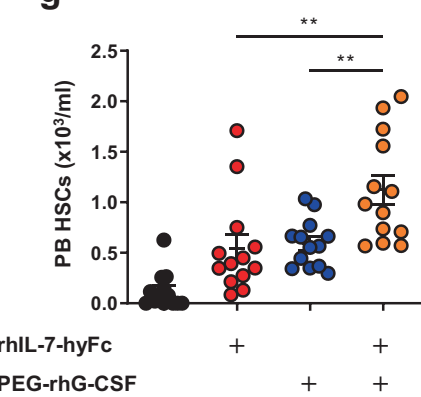

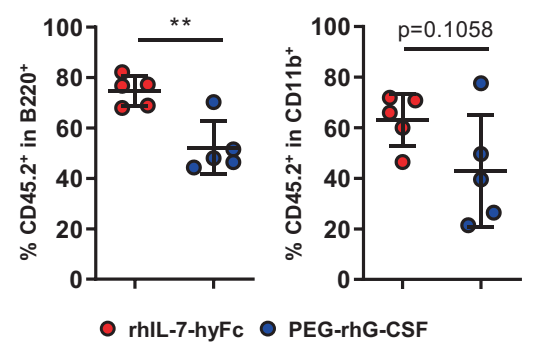

4 rhlL-7-hyFc $(0.5 \mathrm{mg} / \mathrm{kg}$, s.c. $)$ or PEG-rhG-CSF $(250 \mu \mathrm{g} / \mathrm{kg}$, s.c.

$\mathbf{h}$

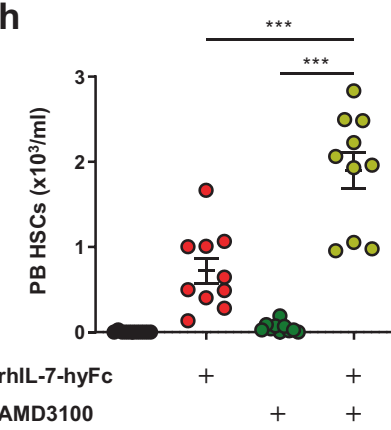

i e
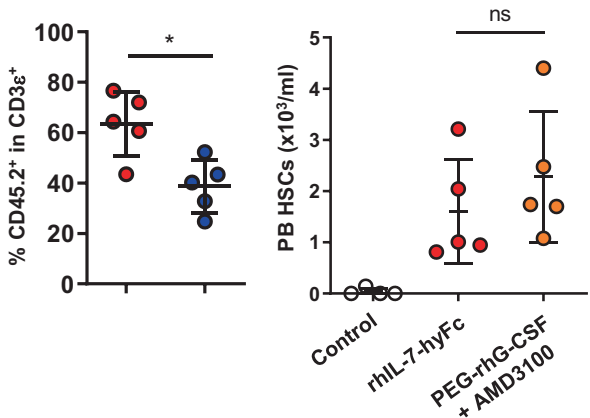

( $(5 \mathrm{mg} / \mathrm{kg}, 1 \mathrm{~h}$ before analysis, s.c. $)$

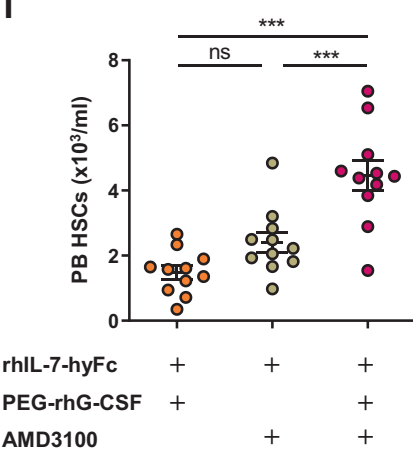

Fig. 2 rhIL-7-hyFc has a superior function for HSC mobilization than G-CSF and synergizes with G-CSF and AMD3100. a, b Mobilization of HSCs by a single dose of rhIL-7-hyFc $(2.5 \mathrm{mg} / \mathrm{kg})$ or PEG-rhG-CSF $(250 \mu \mathrm{g} / \mathrm{kg})$, or a repeated dose of rhG-CSF (bidaily $312.5 \mu \mathrm{g} / \mathrm{kg} /$ day for 4 consecutive days). Experimental scheme (a). Representative dot plot and numbers of PB HSCs $(n=10)$ and CFUGM (rhIL-7-hyFc; $n=8$, other groups; $n=7$ ) (b). c, d Competitive reconstituting assay with peripheral blood mononuclear cells (PBMCs) isolated from rhIL-7-hyFc or PEG-rhG-CSF-treated mice $\left(\mathrm{CD} 45.2^{+}\right)$ and $\mathrm{BM}$ competitor cells $\left(\mathrm{CD} 45.1^{+}\right)$. Blood chimerism in leukocytes at 8,12 , and 18 weeks $(\mathbf{c})$ and in lineage cells at 8 weeks $(\mathbf{d})(n=5)$. e Mobilization of HSCs by control, $2.5 \mathrm{mg} / \mathrm{kg}$ rhIL-7-hyFc, or

rhIL-7-hyFc treatment did not reduce expression of HSC retention-associated genes, such as $C x c l 12, S c f$, and Vcaml, in non-hematopoietic niche cells (Supplementary Fig. S4a). However, rhIL-7-hyFc notably reduced expressions of VLA- combination with $250 \mu \mathrm{g} / \mathrm{kg}$ PEG-rhG-CSF and $5 \mathrm{mg} / \mathrm{kg}$ AMD3100 (control; $n=4$, other groups; $n=5$ ). f-i Combined treatment with $0.5 \mathrm{mg} / \mathrm{kg}$ rhIL-7-hyFc, $250 \mu \mathrm{g} / \mathrm{kg}$ PEG-rhG-CSF, and $5 \mathrm{mg} / \mathrm{kg}$ AMD3100. Experimental scheme (f) and numbers of PB HSCs $(\mathbf{g}-\mathbf{i})$ $(n=13,10$, and 11 for $\mathbf{g}, \mathbf{h}$, and $\mathbf{i}$, respectively). Data are pooled from two independent experiments $(\mathbf{b}, \mathbf{g - i}$, mean \pm SEM) and are representative of 2 or 3 independent experiments $(\mathbf{c}-\mathbf{e}$, mean $\pm \mathrm{SD}$ ). $P$ values were determined by one-way ANOVA with Tukey's multiple comparison for (b), (e), (g-i), two-way ANOVA with Bonferroni's multiple comparison for (c), and unpaired $t$ test for $(\mathbf{d})$. " $n$ " indicates the sample number. $* P<0.05, * * P<0.01, * * * P<0.001$.

4, but not CXCR4 or c-Kit, in HSCs in a pro-B celldependent manner (Supplementary Fig. S4b, c).

We next sought to compare the efficacy of IL-7-induced mobilization to G-CSF, a standard agent used in clinics to 
mobilize HSCs [2]. Two types of G-CSF were used: a single dose of long-acting pegylated rhG-CSF (PEG-rhGCSF), and repeated doses of rhG-CSF (Fig. 2a). All treatments downregulated BM cellularity but upregulated blood and splenic cell counts (Supplementary Fig. S5a, c, g). The single dose of rhIL-7-hyFc significantly increased blood HSPC and CFU-GM levels compared to those induced following PEG-rhG-CSF and rhG-CSF treatment (Fig. 2b, Supplementary Fig. S5b). Mobilized HSPCs by rhIL-7hyFc were also detected in the spleen and their levels were slightly higher than that by G-CSF (Supplementary Fig. S5h-j). As previously observed in Supplementary Fig. S1a-c, rhIL-7-hyFc dropped HSPC and CFU-GM levels in the BM (Supplementary Fig. S5d-f). By contrast, G-CSF administrations reduced HSCs but maintained LSK cells in the BM, leading to CFU-GM counts comparable to control. This difference may result from the G-CSFmediated HSPC expansion [11], which was not seen in rhIL-7-hyFc treatment. Because the mobilization efficacy of two G-CSF types was comparable (Fig. 2b, Supplementary Fig. S5b), we used long-acting PEG-rhG-CSF for subsequent experiments. We next evaluated the repopulating capacity of mobilized HSCs following rhIL-7-hyFc and PEG-rhG-CSF treatment, and observed that PBMCs isolated from rhIL-7-hyFc-treated mice had higher reconstituting activity, compared with PBMCs from PEG-rhGCSF-treated mice (Fig. 2c, d). In clinical practice, G-CSF is often combined with AMD3100 to improve mobilization efficiency in patients who displayed insufficient mobilization with G-CSF treatment alone [3]. Interestingly, our data showed that single rhIL-7-hyFc treatment mobilized HSCs to levels similar to those after combined PEG-rhG-CSF and AMD3100 treatments (Fig. 2e), indicating a superior function of rhIL-7-hyFc compared to G-CSF.

To expand the clinical applicability of rhIL-7-hyFc, we evaluated whether the level of HSC mobilization by rhIL-7hyFc could be enhanced with addition of G-CSF and AMD3100 (Fig. 2f). Injecting both rhIL-7-hyFc and PEGrhG-CSF resulted in increased blood HSC concentrations compared to single treatments (Fig. 2g, Supplementary Fig. S6a). Combined administration of rhIL-7-hyFc and AMD3100 also showed a synergistic effect compared to rhIL-7-hyFc or AMD3100 alone (Fig. 2h, Supplementary Fig. S6c). Interestingly, the triple combination of rhIL-7hyFc, PEG-rhG-CSF, and AMD3100 showed a further increase compared to the double treatments of rhIL-7-hyFc and PEG-rhG-CSF, or rhIL-7-hyFc and AMD3100 (Fig. 2i, Supplementary Fig. S6e). Collectively, our data showed that the combination of rhIL-7-hyFc with G-CSF and AMD3100 synergistically enhanced HSC mobilization. Consistent with previous report [12], G-CSF treatment reduced pro-B cell number in the BM (Supplementary Fig. S6b). However, the combination of rhIL-7-hyFc with
G-CSF preserved pro-B cells in the BM, implying a rescue of B-lymphopoiesis (Supplementary Fig. S6b). rhIL-7-hyFc also upregulated pro-B cells in the $\mathrm{BM}$ along with AMD3100 treatment (Supplementary Fig. S6d).

In this study, we propose rhIL-7-hyFc (GX-I7, NT-I7, efineptakin-alfa) as a new agent that mobilizes HSCs from the BM into the PB after a single treatment. rhIL-7-hyFc promotes HSC mobilization indirectly since HSCs do not express IL-7R [9]. In addition, rhIL-7-hyFc does not regulate crucial interactions between HSCs and their niches, such as CXCR4/CXCL12 and c-Kit/SCF, which are the main targets of conventional mobilization agents [13]. Instead, our data suggests that IL-7R-expressing pro-B cells are critical for HSC mobilization. Interestingly, rhIL-7-hyFc treatment downregulated VLA-4 on BM HSCs in a pro-B cell-dependent manner. Since the VLA-4/VCAM-1 interaction is known to regulate HSC retention in the niche [14], IL-7-mediated reduction of VLA-4 may play a role in HSC mobilization. Notably, pro-B cells reside in perisinusoidal regions where HSCs co-locate, and express similar niche molecules such as CXCR4, c-Kit, and VLA-4 [10, 13, 15]. Thus, we are tempting to speculate the possibility that expanded pro-B cells by rhIL-7-hyFc may competitively occupy the niche, blocking retention interactions for HSCs to induce the mobilization.

Gene transcription profile revealed that a particular myeloid progenitor (PreGM) in the BM also expressed IL-7R (BloodSpot at www.bloodspot.eu). However, BM total myeloid progenitors (MPs), which contain PreGMs, did not express IL-7R in protein levels (Supplementary Fig. S2a) and showed a significant reduction after rhIL-7-hyFc treatment (Supplementary Fig. S1e), indicating MPs are not required to stem cell mobilization by rhIL-7-hyFc. IL-7 treatment is also known to indirectly promote myelopoiesis and erythropoiesis by induction of multiple cytokines such as GM-CSF, IL-3, and Flt3 ligand [6]. It leads us to hypothesize that expanding pro-B cells and other IL-7R-expressing cells, especially activated $\mathrm{T}$ cells, by rhIL-7-hyFc could express factors such as cytokines in the BM that may regulate HSC mobilization. Further studies remain to address those questions.

Phase 1 clinical study proved that rhIL-7-hyFc was safe and well-tolerated in healthy subjects (NCT02860715) [8]. Transient injection site reactions, which were resolved spontaneously, were reported but no serious adverse effects were observed. For the first time, we demonstrated an increase in $\mathrm{CD}_{3} 4^{+}$HSCs in the PB of healthy volunteers after rhIL-7-hyFc treatment. Injected dose of $60 \mu \mathrm{g} / \mathrm{kg}$ in humans (mouse equivalent dose: $0.75 \mathrm{mg} / \mathrm{kg}$ ) is 3.3 -fold lower than the optimal dose used in mice, so future clinical studies are needed to examine optimal and effective regimens. In addition, whether the mechanism by which IL-7 mobilizes BM HSCs in mice is similar to that in humans remains to be determined. Nonetheless, owing to the 
distinct mode of action to mobilize HSCs, our findings suggest rhIL-7-hyFc as a mobilizing agent and synergistic partner for G-CSF and AMD3100 in humans.

In conclusion, we demonstrated that a single dose administration of rhIL-7-hyFc mobilized multipotent long-term HSCs, mediated through IL-7R responses in BM pro-B cells in mice. rhIL-7-hyFc mobilized higher numbers of BM HSCs than G-CSF, and its efficacy was further augmented by cotreatments with G-CSF and AMD3100. Notably, a single rhIL-7-hyFc injection induced $\mathrm{CD} 34^{+}$stem cell mobilization in humans. Our results identify rhIL-7-hyFc as a promising agent for HSC mobilization, working in synergy with conventional mobilizing agents, G-CSF and AMD3100.

Acknowledgements This work was supported by a National Research Foundation of Korea (NRF) grant (2017R1A5A1015366), the Bio \& Medical Technology Development Program of the NRF (2017M3A9C8033570 funded by the Korean government (MSIT)), and the BK21 funded by the Ministry of Education, Republic of Korea (4120200313623). This work was supported by the funding from Genexine, Inc., Republic of Korea and NeoImmuneTech, Inc., USA. We thank You-Me Kim (Korea Advanced Institute of Science and Technology) for providing Mb1-Cre mice.

Author contributions SK, Y-MK, and S-WL conceived the study, designed experiments, and wrote the manuscript. SK, Y-MK, HK, YWK, and SP performed experiments and SK and Y-MK analyzed data. S-IY, DC, and YCS provided rhIL-7-hyFc and human samples. All authors reviewed and approved the manuscript.

\section{Compliance with ethical standards}

Conflict of interest S-IY and YCS are employees of Genexine, Inc., and DC is an employee of NeoImmuneTech, Inc. Both companies provided rhIL-7-hyFc for this study. S-WL received research funding from Genexine, Inc. and NeoImmuneTech, Inc. The other authors declare no competing financial interests.

Publisher's note Springer Nature remains neutral with regard to jurisdictional claims in published maps and institutional affiliations.

Open Access This article is licensed under a Creative Commons Attribution 4.0 International License, which permits use, sharing, adaptation, distribution and reproduction in any medium or format, as long as you give appropriate credit to the original author(s) and the source, provide a link to the Creative Commons license, and indicate if changes were made. The images or other third party material in this article are included in the article's Creative Commons license, unless indicated otherwise in a credit line to the material. If material is not included in the article's Creative Commons license and your intended use is not permitted by statutory regulation or exceeds the permitted use, you will need to obtain permission directly from the copyright holder. To view a copy of this license, visit http://creativecommons. org/licenses/by/4.0/.

\section{References}

1. Holtick U, Albrecht M, Chemnitz JM, Theurich S, ShimabukuroVornhagen A, Skoetz N, et al. Comparison of bone marrow versus peripheral blood allogeneic hematopoietic stem cell transplantation for hematological malignancies in adults - a systematic review and meta-analysis. Crit Rev Oncol Hematol. 2015;94:179-88.

2. Duong HK, Savani BN, Copelan E, Devine S, Costa LJ, Wingard JR, et al. Peripheral blood progenitor cell mobilization for autologous and allogeneic hematopoietic cell transplantation: guidelines from the American Society for Blood and Marrow Transplantation. Biol Blood Marrow Transpl. 2014;20:1262-73.

3. Giralt S, Costa L, Schriber J, Dipersio J, Maziarz R, McCarty J, et al. Optimizing autologous stem cell mobilization strategies to improve patient outcomes: consensus guidelines and recommendations. Biol Blood Marrow Transpl. 2014;20:295-308.

4. Grzegorzewski K, Komschlies KL, Mori M, Kaneda K, Usui N, Faltynek CR, et al. Administration of recombinant human interleukin-7 to mice induces the exportation of myeloid progenitor cells from the bone marrow to peripheral sites. Blood. 1994;83:377-85.

5. Grzegorzewski KJ, Komschlies KL, Jacobsen SE, Ruscetti FW, Keller JR, Wiltrout RH. Mobilization of long-term reconstituting hematopoietic stem cells in mice by recombinant human interleukin 7. J Exp Med. 1995;181:369-74.

6. Aiello FB, Keller JR, Klarmann KD, Dranoff G, Mazzucchelli R, Durum SK. IL-7 induces myelopoiesis and erythropoiesis. J Immunol. 2007;178:1553-63.

7. Choi YW, Kang MC, Seo YB, Namkoong H, Park Y, Choi DH, et al. Intravaginal administration of Fc-fused IL7 suppresses the cervicovaginal tumor by recruiting HPV DNA vaccine-induced CD8 T cells. Clin Cancer Res. 2016;22:5898-908.

8. Lee SW, Choi D, Heo M, Shin EC, Park SH, Kim SJ, et al. hIL-7hyFc, a long-acting IL-7, increased absolute lymphocyte count in healthy subjects. Clin Transl Sci. 2020;13:1161-9.

9. Mackall CL, Fry TJ, Gress RE. Harnessing the biology of IL-7 for therapeutic application. Nat Rev Immunol. 2011;11:330-42.

10. Fistonich C, Zehentmeier S, Bednarski JJ, Miao R, Schjerven H, Sleckman BP, et al. Cell circuits between B cell progenitors and IL-7(+) mesenchymal progenitor cells control B cell development. J Exp Med. 2018;215:2586-99.

11. Walter D, Lier A, Geiselhart A, Thalheimer FB, Huntscha S, Sobotta MC, et al. Exit from dormancy provokes DNA-damageinduced attrition in haematopoietic stem cells. Nature. 2015;520:549-52.

12. Winkler IG, Bendall LJ, Forristal CE, Helwani F, Nowlan B, Barbier V, et al. B-lymphopoiesis is stopped by mobilizing doses of G-CSF and is rescued by overexpression of the anti-apoptotic protein Bcl2. Haematologica. 2013;98:325-33.

13. Crane GM, Jeffery E, Morrison SJ. Adult haematopoietic stem cell niches. Nat Rev Immunol. 2017;17:573-90.

14. Rettig MP, Ansstas G, DiPersio JF. Mobilization of hematopoietic stem and progenitor cells using inhibitors of CXCR4 and VLA-4. Leukemia. 2012;26:34-53.

15. Balzano M, De Grandis M, Vu Manh TP, Chasson L, Bardin F, Farina A, et al. Nidogen-1 contributes to the interaction network involved in Pro-B cell retention in the peri-sinusoidal hematopoietic stem cell niche. Cell Rep. 2019;26:3257-71 e3258. 